\title{
Platform Pricing in Matching Markets
}

\author{
Maarten Goos, Patrick Van Cayseele and Bert Willekens*
}

November 18, 2013

\begin{abstract}
Existing models of two-sided markets explain why platforms charge different prices between buyers and sellers. Generally, the platform will subsidize participation on a side of the market the higher is that side's positive cross-side externality to users on the other side of the market. However, in matching markets there also exists a negative own-side congestion externality that the platform internalizes by taxing users for its presence. Assuming a monopoly platform pricing model, the first contribution of this paper is to show that these positive cross-side and negative own-side externalities can be summarized by the matching elasticity derived from a general matching function that captures the efficiency of the platform's matching technology. The platform charges a lower price to a side of the market the higher is that side's matching elasticity. The second contribution of this paper is to show that the platform's pricing strategy only partially internalizes the efficiency of its matching technology, compared to the social optimum. In particular, we discuss the possibility that a monopoly matchmaker sets too high a price on the low-price side of the market and too low a price on the high-price side of the market, resulting in insufficient inequality in prices between sides of the platform.
\end{abstract}

Keywords: platform pricing, matching markets

\footnotetext{
• Goos: University of Leuven, maarten.goos@kuleuven.be. Van Cayseele: University of Leuven, patrick.vancayseele@kuleuven.be. Willekens: University of Leuven, bert.willekens@kuleuven.be. We gratefully acknowledge Jan Bouckaert, Carlos Cañón, Renato Gomes, Joep Konings, Alan Manning, Andras Niedermayer, Anna Salomons, Maarten Pieter Schinkel, Jo Van Biesebroeck and numerous seminar and conference participants for useful conversations and discussions. Special thanks go to Glen Weyl for numerous valuable suggestions and detailed comments on previous drafts of the paper. Goos and Van Cayseele acknowledge the University of Leuven Program Financing/Center of Excellence and Willekens the Research Foundation Flanders (Scholarship DME-C1972-ASP/10) for financial support.
} 


\section{Introduction}

Increasing volumes of transactions in matching markets happen through private platforms. Characteristic to these matchmaking platforms is the existence of network externalities. For example, the probability for an individual worker to find a job through an online job board decreases when there are more job-seekers and fewer vacancies. Similarly, a realtor may find it difficult to sell one's home when rivalry among sellers is fierce. In such settings where the matching technology is characterized by important own-side and cross-side externalities, what prices should private platforms charge on both sides of the market and what implications do these externalities entail for welfare?

Platforms charge different prices between sides of the market (see, among others, Caillaud and Jullien (2003), Rochet and Tirole (2003, 2006), Armstrong (2006), Weyl (2010)). Generally, the platform's price to one side of the market will be lower the larger is that side's positive cross-side externality to users on the other side of the market. For example, when one side of the market only differs in that there are more users, the platform will charge this side a lower price because it is easier for users on the other side to trade. However, matching markets are also characterized by a negative own-side congestion externality. For example, the realization of a worker-firm match implies that this vacancy is no longer available to other job-seekers and that this worker is no longer employable at other firms.

To formally analyze the different incentives that a matchmaking platform has in its pricing behavior, this paper first introduces a general matching function - a concept wellknown from the labor economics literature (see, for example, Petrongolo and Pissarides (2001) for a review) - that captures the platform's matching technology. The matching technology is said to be more efficient for a side of the market when the negative own-side externality - which we express in absolute value throughout the paper - is small and the positive cross-side externality is large. We show that the platform's matching efficiency for a side of the market can be meaningfully summarized by that side's matching elasticity, defined as the percentage increase in total matches for a percentage increase in own-side participation. That is, a smaller negative own-side and larger positive cross-side externality 
- or a more efficient matching technology - implies a higher matching elasticity for that side of the platform.

The first contribution of this paper is to show that the optimal price charged by a monopoly matchmaker to a side of the market is lower when that side's matching elasticity is higher. The intuition for this result is simple. A higher matching elasticity results from a smaller negative own-side and a larger positive cross-side externality. Because this gives the monopoly matchmaker less of an incentive to tax the negative own-side effect and more of an incentive to subsidize the positive cross-side externality, its price will be lower. Consistently, we show that in a symmetric setting in which two sides differ only in their matching elasticities, the monopoly matchmaker charges a lower price to the side that has the highest matching elasticity. The results are illustrated for a monopoly matchmaker that uses a listing technology: one side of the market posts advertisements and the other side searches through these ads. We show that the platform sets prices such that the searching side of the market has the highest matching elasticity and hence is charged the lowest fee.

The second contribution of this paper is to show that a monopoly matchmaker only partially internalizes the efficiency of its matching technology compared to the incentives of a social planner. We show that a higher matching elasticity leads to a larger difference between the private platform's and socially optimal price. The intuition for this is the following. A higher matching elasticity decreases the profit maximizing price which increases participation by marginal users. However, these marginal users value the platform's service less than the average user. This heterogeneity in user types gives the private platform an incentive to discourage participation by charging a higher price compared to a social maximizer that internalizes the matching externalities through their average and not marginal user valuations. In addition to this, we derive a relationship between the matching technology and price distortions between sides of the platform. In particular, we show that a monopoly matchmaker could set too low a price on the highprice side of the market and too high a price on the low-price side of the market, resulting in inequality in prices between sides of the platform that is too low from a social point of 
view. We illustrate this case assuming realistic parameter values for lognormally distributed heterogeneity in user types and a constant returns to scale matching function.

In the literature, various other contributions have recently been made on the topic of optimal pricing by matching platforms. Closest related is Chen and Huang (2012) who consider a specific matching technology where sellers post the price for their goods to attract buyers and buyers choose sellers. As is the case here, the platform's optimal price depends on the platform's matching technology. Although their framework is in many ways richer than ours (e.g. we do not analyze the possibility that platform fees are passed through by sellers in the price of a good), the assumed matching process is specific and therefore does not allow for the derivation of the relationship between the platform's optimal prices and its matching technology as generally. For example, the result of Chen and Huang (2012) that the seller-side of the market is never subsidized by a private platform is confirmed by the present study. We show that for a listing platform the matching elasticity of the seller-side is indeed lower than that of the buyer-side and hence the seller-side is charged the highest fee. Furthermore, we show that this price asymmetry is likely to be insufficient compared to the social optimum.

Also closely related is the study of Niedermayer and Shneyerov (forthcoming) who analyze optimal platform pricing in a dynamic random matching model with buyer-seller bargaining. These authors show that under a symmetric matching technology, asymmetric bargaining weights results in asymmetric optimal platform fees and suggest that the presence of a monopoly intermediary in a search market can be welfare enhancing. The present paper has exogenous valuations for buyers and sellers and ignores certain bargaining issues to focus on the market distortions that potentially emerge from an asymmetric matching technology.

Also, Damiano and Li (2007, 2008) explore the sorting role of optimal pricing by a private monopolist and by duopolists under user "quality” differentiation. In contrast, we abstract from complementarities between user types such that sorting of users plays no role and we do not allow the platform to price discriminate within user groups. Our focus is on 
the network externalities linked to the matching technology, a feature not explicitly analyzed in Damiano and Li (2007, 2008).

Finally, Ellison and Fudenberg (2003) propose a general framework to analyze how the joint presence of cross- and own-side network externalities affects the market participation decision of two types of user groups. They do not explicitly analyze, however, the optimal pricing behavior of platforms. Belleflamme and Toulemonde (2009), among others, do analyze the impact of both network effects on optimal platform behavior, but not in a setting of one-to-one matching as in the present paper.

The remainder of the paper is organized as follows. Section 2 presents the simplest possible framework of a monopoly matchmaker where network externalities are captured by a matching function that generally characterizes the platform's matching technology. Section 3 shows how the platform's optimal prices depend on the platform's matching technology, and Section 4 examines implications for welfare. Section 5 concludes.

\section{Framework}

\subsection{User participation}

Consider a platform that connects two types of user groups $I=A, B$. When users on side $I$ participate they have a probability $0 \leq m^{I} \leq 1$ of being matched, assumed to be the same for all users on the same side but allowed to differ across sides - these matching probabilities are further characterized in the next subsection. Users on side $I$ are heterogeneous in two dimensions: $V^{I} \in \mathbb{R}$ denotes the valuation of an $I$-side user when matched and $Z^{I} \in \mathbb{R}$ when unmatched. For example, the match valuation $V^{I}$ could be the wage a job-seeker expects to get or the price a home-seller expects to receive, net of any costs for searching the platform. The outside option $Z^{I}$ could be the job-seeker's unemployment benefit or the mortgage cost for the home-owner (in which case $Z^{I}$ will be negative).

The platform charges a flat fee for the matching service, which is allowed to differ across the two sides of the market, but is the same to all participants within sides. The price 
is modeled as a per-match fee $p^{I}$ which is paid by participants conditional on being matched. Note that the price can equivalently be modeled as a participation fee $P^{I}$ which is paid by users when they enter the platform by simply substituting for $p^{I}=P^{I} / m^{I}$ in all expressions below. We prefer, however, to display the analysis in terms of per-match fees because it simplifies notation. Further note that the equivalence between the two fee types might not necessarily hold in practice. For example, it could be costly to monitor matching outcomes such that the platform would prefer charging participation over per-match fees. Appendix A discusses the assumptions underlying the equivalence in greater detail and elaborates on cases for which it no longer holds.

In the setting described, expected utility for a user on side $I$ of the platform equals:

$$
U^{I}=\left(V^{I}-p^{I}\right) m^{I}+Z^{I}\left(1-m^{I}\right)
$$

Expected utility net of the outside option, defined as $u^{I} \equiv U^{I}-Z^{I}$, can then be written as:

$$
u^{I}=\left(v^{I}-p^{I}\right) m^{I}
$$

in which $v^{I} \equiv V^{I}-Z^{I}$ is defined as the net match valuation. From equation (2) it follows that an $I$-side user will participate if his net match valuation is positive, i.e. when $u^{I} \geq 0{ }^{1}$

Finally, assume that the net match valuation $v^{I}$ is distributed by a twice continuously differentiable distribution function $F^{I}($.$) and density function f^{I}($.$) that are public$ information. For a mass of potential participants $n^{I}$, the number of participating users on side $I$ of the platform is given by: ${ }^{2}$

$$
N^{I}=n^{I}\left(1-F^{I}\left(p^{I}\right)\right)
$$

\footnotetext{
${ }^{1}$ Note that the participation decision only depends on a single source of user heterogeneity, i.e. a user's net match valuation $v^{I}$. This simplification follows from the assumptions that users are risk neutral and that they differ in their outside options rather than in their fixed benefits of using the platform as is assumed in, among others, Armstrong (2006), Rochet and Tirole (2006) and Weyl (2010).

${ }^{2}$ Note that we deviate from the classic platform pricing models by not normalizing the mass of potential users to unity on both sides of the market. The reason for this is that most specifications of the matching function are only sensible when thinking about a "number" rather than a "fraction" of participating users.
} 


\subsection{The matching function and matching elasticities}

If $N^{A}$ users participate on side $A$ and $N^{B}$ users participate on side $B$ of the platform, we assume that the total number of matches is given by the well-known matching function $M=M\left(N^{A}, N^{B}\right)$. The advantage of introducing this matching function is that it allows us to account for the efficiency of the matching technology without having to make explicit the imperfections in the matching process. We also assume that the platform is a random matchmaker such that all participants on the same side have the same probability of being matched:

$$
m^{I}=M / N^{I}
$$

As is standard in the matching literature, the matching function $M\left(N^{A}, N^{B}\right)$ is assumed to be (i) twice continuously differentiable; (ii) weakly increasing; (iii) weakly concave; and to have that (iv) $M\left(N^{A}, 0\right)=M\left(0, N^{B}\right)=0$; (v) $M \leq \min \left[N^{A}, N^{B}\right]$. Under these weak regularity conditions, it is easy to show that the match probability $m^{I}=M\left(N^{I}, N^{J}\right) / N^{I}$ (with $J$ the other side than $I$ ) is weakly decreasing in own-side participation $N^{I}$ which captures a negative own-side externality, and weakly increasing in cross-side participation $N^{J}$ which captures a positive cross-side externality.

A useful way to summarize the negative own-side and positive cross-side externalities in the matching technology is through the matching elasticity for side $I$ :

$$
\phi^{I} \equiv \frac{\partial M}{\partial N^{I}} \frac{N^{I}}{M}
$$

with $0 \leq \phi^{I} \leq 1$. The matching elasticity for side $I$ is defined as the percentage increase in the total number of matches for a percentage increase in own-side participation. Importantly, there is a one-to-one relationship between the negative own-side and positive cross-side externalities and the matching elasticity. To see this, note that the negative ownside externality per-match is given by:

$$
\left|N^{I} \frac{\partial m^{I}}{\partial N^{I}} \frac{1}{m^{I}}\right|=1-\phi^{I}
$$


which is the absolute value of the sum across $N^{I}$ participants (which gives the term $N^{I}$ ) of the decrease in their match probability when another $I$-side user enters the platform (which gives the term $\partial m^{I} / \partial N^{I}$ ) rescaled on a per-match base (which gives the term $1 / \mathrm{m}^{I}$ ). Similarly, the positive cross-side externality per-match is the sum across $N^{J}$ participants of the increase in their match probability when another $I$-side user enters the platform:

$$
N^{J} \frac{\partial m^{J}}{\partial N^{I}} \frac{1}{m^{I}}=\phi^{I}
$$

The intuition of equations (6) and (7) is straightforward. Equation (6) shows that the matching elasticity is decreasing in the negative own-side externality. The reason for this is that an additional participant on side $I$ decreases the matching probability for all $I$-side users. This effect is smaller if additional users are more easily matched, i.e. if the matching elasticity is larger. Similarly, equation (7) shows that the matching elasticity is increasing in the positive cross-side externality because an additional user on side $I$ leads to an increase in the matching probability for $J$-side users that is higher the more efficient the platform is at matching $I$-side users.

\subsection{Private monopoly platform}

We assume that there is a private monopoly platform that sets per-match fees to both sides of the platform in a first stage. In the second stage potential users on both sides simultaneously decide to participate. In a third stage, matched users pay the per-match fee to the platform. In this setting, the monopoly matchmaker will choose per-match fees $p^{A}$ and $p^{B}$ to maximize profits given by:

$$
\pi\left(p^{A}, p^{B}\right)=\left(p^{A}+p^{B}\right) M\left(N^{A}, N^{B}\right)
$$

subject to equation (3). Also note that we have assumed for simplicity that there are no costs for operating the platform. 


\section{Analysis}

\subsection{Equilibrium prices}

Proposition 1 follows from the first-order conditions of the profit maximization problem, in which $\mu^{I} \equiv\left(1-F^{I}\left(\widetilde{v^{I}}\right)\right) / f^{I}\left(\widetilde{v^{I}}\right)$ is the inverse hazard rate of demand on side $I$ and $\widetilde{v^{I}}$ is the net match valuation of the marginal $I$-side participant who is indifferent between participating or not such that $\widetilde{v^{I}} \equiv p^{I}:^{3}$

Proposition 1. At the optimal allocation, the per-match fee a private monopoly platform charges on each side $I=A, B(J \neq I)$ is:

$$
p^{I}=\mu^{I}+\widetilde{v^{I}}\left(1-\phi^{I}\right)-\widetilde{v^{J}} \phi^{I}
$$

The first term on the right-hand side of equation (9) is the inverse hazard rate of demand, which is the classic Cournot (1838) measure of monopoly market power. The second term on the right-hand side shows how the platform internalizes the per-match negative own-side externality by taxing $I$-side users. This tax is larger when the matching elasticity is smaller, i.e. when the platform's matching technology is less efficient because the negative own-side externality is stronger. The final term on the right-hand side of equation (9) shows how the platform subsidizes the positive cross-side externality. This subsidy is increasing in the matching elasticity because a more efficient matching technology exists when an additional I-side user increases the matching probability of any $J$-side user by more.

In sum, we show that the platform's matching technology - characterized by negative own-side and positive cross-side externalities - can be meaningfully summarized by the matching elasticity and indicate that the price a monopoly matchmaker charges on any side of the platform is decreasing in that side’s matching elasticity.

\footnotetext{
${ }^{3}$ It is well-known from the platform pricing literature that in the described optimization problem, induced by the presence of network externalities, there is equilibrium multiplicity because of user coordination failures. Appendix B discusses various approaches proposed by Caillaud and Jullien $(2001,2003)$ and Weyl $(2010)$ of how the platform can uniquely establish its preferred equilibrium.
} 


\subsection{Asymmetric pricing between sides of the platform}

So far we have analyzed what determines the optimal price on a given side of the platform. An interesting question is what this implies for price asymmetries between sides of the platform. Using equation (9) and the fact that $\widetilde{v^{I}} \equiv p^{I}$ and $\widetilde{v^{J}} \equiv p^{J}$, we get that:

$$
p^{A}+p^{B}=\frac{\mu^{A}}{\phi^{A}}=\frac{\mu^{B}}{\phi^{B}} \text { or that } \frac{\phi^{A}}{\phi^{B}}=\frac{\mu^{A}}{\mu^{B}}
$$

where the inverse hazard rates are given by $\mu^{I} \equiv\left(1-F^{I}\left(p^{I}\right)\right) / f^{I}\left(p^{I}\right)$ for $I=A, B$. For example, assume that $\phi^{A}>\phi^{B}$ and that $\mu^{I}$ is decreasing in $p^{I}$ for $I=A, B$. If the platform's technology is such that it is better at matching side- $A$ than side- $B$ users such that $\phi^{A}>\phi^{B}$, we must have that $\mu^{A}>\mu^{B}$. And if these hazard rates are decreasing in their prices such that $p^{A}<p^{B}$, the platform will charge a lower price to the side that has the highest matching elasticity. ${ }^{4}$

Whether $\mu^{I}$ is increasing or decreasing in $p^{I}$ depends on the distribution of net match valuations. ${ }^{5}$ To summarize this, assume for simplicity that net match valuations are symmetrically distributed and that the matching elasticities are constant. Then from Proposition 1 and equation (10) it follows that:

Corollary 1. Assume symmetrically distributed net match valuations $\left(F^{A}()=.F^{B}().\right)$ and a constant elasticity matching function ( $\phi^{A}$ and $\phi^{B}$ constant). Then, for a decreasing inverse hazard rate of demand, we have that:

$$
p^{A}<p^{B} \Leftrightarrow \phi^{A}>\phi^{B}
$$

The result reverses for an increasing hazard rate.

\footnotetext{
${ }^{4}$ Also note that equation (10) is consistent with Rochet and Tirole (2003) who assume that each user on one side can interact with all the users on the other side. This implies a 'matching elasticity' of unity on each side of the market. Consequently, they show that the optimal price structure satisfies $p^{A}+p^{B}=\mu^{A}=\mu^{B}$ or that price asymmetries can arise because of differences in underlying preferences between both sides of the platform. However, equation (10) shows that asymmetric prices can also be explained by the properties of the matching technology under which the platform operates even if preferences are symmetrically distributed.

${ }^{5}$ Fabinger and Weyl (2012) provide a formal discussion on the properties of demand and show that for the majority of distribution classes the hazard rate is decreasing in price.
} 


\subsection{An application: a listing service platform}

In Corollary 1 we have assumed that matching elasticities are constant, i.e. independent of the chosen allocation by the platform. However, externalities induced by a marginal participant on either side might vary with the amount of users already present on both sides of the platform. For example, the externalities induced by a marginal worker that enters a job search engine could be very different whether that worker is the 10th or 1000th user to enter the platform. To explore this, one needs to go further and model the microfoundations of the matching function which is what we do in this subsection. More specifically, we reinterpret a familiar microfoundation of the matching function - "urnball” matching - as the matching technology of a listing service platform. The conclusion is that the intuition derived above remains valid even if the matching elasticities are no longer constants. Also then the side of the platform that has the higher matching elasticity is charged a lower price.

Consider a market in which a monopoly platform serves as a matchmaker by offering a listing service. Sellers participate by posting an advertisement for their goods on the platform and buyers search across sellers. We refer to users on the posting-side as firms (side $A$ ) who post job vacancies and the searching-side as workers (side $B$ ), but one can similarly think of $A$ as sellers and $B$ as buyers of any good or service. Each participating firm lists a single vacancy and each participating worker randomly applies to a single vacancy. So, some firms may receive no applications while others may receive many. It is assumed that those firms receiving more applications randomly select one, such that some workers will remain unmatched. In this setting, it is the presence of coordination failures among workers that characterizes the platform's matching technology and externalities.

The matching process can be formally analyzed as follows. Denote the number of firms that list on the platform by $N^{A}>1$. Given a probability $1 / N^{A}$ that a firm receives an application from a given worker, the probability of not receiving an application from that worker is $\left(1-1 / N^{A}\right)$. Denoting the number of workers that search on the list of vacancies by $N^{B}>1$, the probability that a firm ends up without any worker is then given by: 


$$
\left(1-1 / N^{A}\right)^{N^{B}} \approx \exp \left(-N^{B} / N^{A}\right)
$$

where the approximation holds only if $N^{A}$ is sufficiently large. Hence, the probability that a given firm will be matched to a worker is approximated by $1-\exp \left(-N^{B} / N^{A}\right)$.

If there are $N^{A}$ firms listing on the platform, the expected number of total matches is then given by the following matching function:

$$
M\left(N^{A}, N^{B}\right)=N^{A}\left(1-\exp \left(-N^{B} / N^{A}\right)\right)
$$

It follows from this matching function that the matching elasticities with respect to workers and firms are, respectively:

$$
\phi^{B}=\frac{N^{B}}{N^{A}} \frac{\exp \left(-N^{B} / N^{A}\right)}{1-\exp \left(-N^{B} / N^{A}\right)} \text { and } \phi^{A}=1-\phi^{B}
$$

which are not constants. Moreover, the matching elasticities are not symmetric between both sides of the platform. For example, assuming that $N^{A}=N^{B}$, we get that $\phi^{A}=0.42$ and $\phi^{B}=0.58$. The higher matching elasticity for workers than for firms captures the fact that the listing platform is more efficient at matching workers than firms. One intuition for this is that workers are "active" in this model in the sense that they apply to vacancies taking into account the (expected) behavior of other workers. In contrast, firms are "passive" in the sense that they simply post a vacancy and hope at least one worker applies.

Further assume, for simplicity, that the mass of potential participants is symmetric, $n^{A}=n^{B}$, and sufficiently large. Also assume that the net match valuations $v^{A}$ and $v^{B}$ are both uniformly distributed over the unit interval such that inverse demand on each side $I=A, B$ of the platform is given by $p^{I}=1-N^{I} / n^{I}$. This implies a decreasing inverse hazard rate of the form $\mu^{I}=1-p^{I}$ for $I=A, B$. Solving for profit maximizing prices accounting for the fact that the matching elasticities are no longer constants - gives the following outcomes for the worker side of the platform: $p^{B}=0.47$ and $\mu^{B}=\phi^{B}=0.53$. For the firm side of the platform we get: $p^{A}=0.53$ and $\mu^{A}=\phi^{A}=0.47$. Consequently, the optimal per match fee is lower on the worker side that also has a higher matching elasticity as Corollary 1 suggests. 


\section{Welfare analysis}

\subsection{Socially optimal price setting}

An important question is how the optimal pricing behavior of a private monopoly matchmaker that we discussed in the previous section compares to the outcome of a social planner. Assume a Pigouvian platform that sets prices to maximize its total social value, equal to the sum of aggregate utility of participants on the two sides of the market and the private platform's profits. The maximization problem for the welfare maximizer is then given by:

$$
\max _{p_{\omega}^{A}, p_{\omega}^{B}} \omega=\left(\overline{v^{A}}+\overline{v^{B}}\right) M\left(N^{A}, N^{B}\right)
$$

subject to equation (3) for $I=A, B$ and where:

$$
\overline{v^{I}} \equiv \frac{n^{I}}{N^{I}} \int_{p_{\omega}^{I}}^{\infty} v^{I} f^{I}\left(v^{I}\right) d v^{I}
$$

denotes the average net match valuation of participants on side $I=A, B$. Proposition 2 shows how the social maximizer internalizes the negative own-side and positive cross-side externality in the matching function:

Proposition 2. At the optimal allocation, a Pigouvian platform charges a per-match fee $p^{I}$ on each side $I=A, B(J \neq I)$ that equals:

$$
p_{\omega}^{I}=\overline{v^{I}}\left(1-\phi^{I}\right)-\overline{v^{J}} \phi^{I}
$$

From equation (17) it is clear that the Pigouvian platform also taxes the negative ownside and subsidizes the positive cross-side externality. However, it does so proportional to the average net match valuation of all users participating on the platform, and not the marginal user's match valuation as was the case in Proposition 1. Before turning to the market distortions that result from these diverging incentives, first note that the Pigouvian price can also be written as:

$$
p_{\omega}^{I}=\overline{v^{I}} \phi^{J}-\overline{v^{J}} \phi^{I}+\bar{v}^{I}\left(1-\phi^{I}-\phi^{J}\right)
$$


Equation (18) demonstrates that when the matching technology is characterized by constant returns to scale, the Pigouvian platform will unambiguously subsidize one side of the market and will exactly recover this subsidy from the other side. To see this, note that for $\phi^{A}+\phi^{B}=1, p_{\omega}^{A}=\overline{v^{A}} \phi^{B}-\overline{v^{B}} \phi^{A}$ and $p_{\omega}^{B}=\overline{v^{B}} \phi^{A}-\overline{v^{A}} \phi^{B}$, which are exactly opposites: $p_{\omega}^{A}=-p_{\omega}^{B}$. Which side of the market is subsidized depends on the matching technology, but also on the underlying heterogeneity of net match valuations. This result is further illustrated in the application in subsection 4.4.

\subsection{Market Distortions}

To illustrate the diverging incentives of the private and Pigouvian platform more clearly, we can use equations (9) and (17) to define the market distortion on side $I$ as:

$$
M D^{I} \equiv p^{I}-p_{\omega}^{I}=\mu^{I}+\underbrace{\left(\overline{v^{J}}-\widetilde{v^{J}}\right) \phi^{I}-\left(\overline{v^{I}}-\widetilde{v^{I}}\right)\left(1-\phi^{I}\right)}_{\text {Spence distortion }}
$$

The first term on the right-hand side of equation (19) is the inverse hazard rate of demand the classic Cournot distortion - that captures the market power of the private monopolist. The final two terms on the right-hand side of equation (19) relate to the matching externalities and, following Weyl (2010), can be interpreted as a Spence distortion. ${ }^{6}$

The first term of the Spence distortion is consistent with Weyl (2010) who shows that the diverging incentives of the private and Pigouvian platform to account for positive crossside externalities result in an upward distortion of prices. ${ }^{7}$ By only internalizing the crossside externality at the marginal and not average valuation of cross-side users, the private monopoly platform subsidizes this externality less than what is socially desirable. Consequently, the positive cross-side externality results in too high a price and too little participation. However, the last term in equation (19) shows that when the platform is a

\footnotetext{
${ }^{6}$ This terminology refers to the contribution of Spence (1975), who first pointed out that a monopoly that decides both on price (or quantity) and product quality tends to serve the quality preferences of marginal consumers instead of average consumers as would be optimal from a social point of view. Weyl (2010) revisits this argument for multi-sided platforms, by interpreting the amount of users on one side as a measure of quality of the platform service for users on the other side.

${ }^{7}$ This result unambiguously holds when there is only one source of user heterogeneity in transaction valuations - or net match valuations in our setting - which implies $\overline{v^{I}}>\widetilde{v^{I}}$ is always positive. More generally, it is possible to have a negative Spence distortion also when there are only positive cross-side externalities. For example, Rochet and Tirole (2006) allow for multidimensional heterogeneity in user types and equilibrium in their model could result in a negative Spence distortion. As argued by Weyl (2010) this will be the case if the spread between the average transaction valuation of marginal and infra-marginal users is negative, which will depend on the dominating source of heterogeneity in user types.
} 
matchmaker, there also is a negative own-side externality that leads to a negative term in the Spence distortion. The reason for this is that the monopoly matchmaker taxes the negative own-side externality at the marginal less than average valuation of own-side users. This leads to a price that is less than what is socially desirable and too much participation.

Note that even though there is only one source of user heterogeneity in our setting, the Spence distortion can be downward if the negative own-side effect dominates the positive cross-side effect - a result that cannot be obtained from standard platform models where own-side congestion plays no role. On any side this is more likely to be the case if the matching elasticity is smaller, i.e. when the platform is a less efficient matchmaker on that side of the market. In the next two subsections we explore the relationship between the platform's matching technology and market distortions on both sides of the market in greater detail.

\subsection{Asymmetries in Spence distortions between sides of the platform}

An interesting question is how the Spence distortion relates between sides of the platform and how this relationship depends on the platform's matching technology. This is what we examine in this subsection. We show that when the matching function exhibits decreasing (increasing) returns to scale, the Spence distortion must be negative (positive) on at least one side of the market.

To see this, define $\overline{s^{I}} \equiv \overline{v^{I}}-p^{I}=\overline{v^{I}}-\widetilde{v^{I}}$ as the average per-match surplus of users on side $I=A, B$ of the market and note that (19) can be written as (with $J \neq I$ ):

$$
M D^{I}=\mu^{I}+\overline{s^{J}} \phi^{I}-\overline{s^{I}} \phi^{J}-\overline{s^{I}}\left(1-\phi^{I}-\phi^{J}\right)
$$

For example, assume that the matching function has constant returns to scale, i.e. $\phi^{A}+$ $\phi^{B}=1$. If this is the case, the sign of the Spence distortion on side $A$ coincides with the sign of $\overline{s^{B}} \phi^{A}-\overline{s^{A}} \phi^{B}$ and on side $B$ with $\overline{s^{A}} \phi^{B}-\overline{s^{B}} \phi^{A}$. Consequently, when the Spence distortion is positive on one side, it must be negative on the other side. Equation (20) also provides a more general relationship between Spence distortions on both sides of the market and the platform's matching technology: 
Corollary 2. When the matching function has weakly decreasing returns to scale $\left(\phi^{A}+\right.$ $\left.\phi^{B} \leq 1\right)$, the Spence distortion is weakly negative on at least one side of the market. When the matching function has increasing returns to scale $\left(\phi^{A}+\phi^{B}>1\right)$, the Spence distortion is positive on at least one side of the market.

Finally note that Corollary 2 and equation (20) do not exclude the possibility that the market distortion as a whole is negative. For example, assume that the negative own-side externality on side $B$ of the market is large such that $\phi^{B}$ is small and $\phi^{A}+\phi^{B} \leq 1$. From Corollary 2 we then know that the Spence distortion must be negative on at least one side of the market. Say this is the high-price side $B$ of the market. If on this side the negative Spence distortion is larger in absolute value than the classic distortion resulting from market power, equation (19) shows that the market distortion as a whole will be negative. What this means is that the private monopolist charges the high-price side $B$ too low a price. Moreover, the Spence distortion on the low-price side $A$ of the market will be too high, such that the inequality in market prices between both sides of the market is too low from a social point of view. The next subsection shows this is the case when heterogeneity is lognormally distributed assuming realistic parameter values and when there is a constant returns to scale matching function.

\subsection{An application: lognormal heterogeneity and constant returns to scale matching}

The previous subsection derived a relationship between the platform's matching technology and market distortions in a very general way. For example, it didn't determine on which side of the market the Spence distortion will be negative when the matching function has decreasing or constant returns to scale. And for the side that has the negative Spence distortion, whether the market distortion as a whole is also negative. To answer these type of questions we need to be more specific about the underlying heterogeneity of user types and returns to scale in the matching function. This is what we do in this subsection. 
Assume lognormally distributed net match valuations, $F($.$) , for both workers and$ firms. This could be the case if labor productivity and therefore the wage is lognormally distributed, and firms value productivity and workers value wages. More specifically, assume that net match valuations are lognormally distributed on both sides with mean 10.4 and standard deviation 0.85. We borrow these parameter values from Fabinger and Weyl (2012), who obtain these values by calibrating the lognormal distribution to the 2011 US yearly income distribution. Also assume a constant elasticity matching function homogeneous of degree 1 such that $\phi^{A}+\phi^{B}=1$.

From Corollary 1 we know that if $\phi^{B}>\phi^{A}$ we must also have that $p^{B}<p^{A}$ when the inverse hazard rate of demand is decreasing in price, which can easily be shown to be the case in the present example. Moreover, from equation (20) it follows that the sign of the Spence distortion on the worker-side coincides with the sign of $\overline{s^{A}} \phi^{B}-\overline{s^{B}} \phi^{A}$ and on the firm-side with $\overline{s^{B}} \phi^{A}-\overline{s^{A}} \phi^{B}$. So if the Spence distortion is negative on one side of the market, it must be positive on the other. To see on which side it is positive and on which side it is negative, we need to solve the model and that is what we do next.

Figure 1 illustrates the model's optimal prices and distortions on both sides of the market. The horizontal axes depict the matching elasticity on the worker-side ranging from 0.5 to 1 , and hence the matching elasticity on the firm-side ranging from 0.5 to zero given that $\phi^{B}=1-\phi^{A}$. In other words, Figure 1 shows what happens to equilibrium prices and distortions on both sides of the market when the efficiency of the matching technology becomes more asymmetric in favor of the worker-side of the market.

\section{[Figure 1]}

The top-left panel of Figure 1 shows that the optimal private price for workers goes down and for firms goes up when $\phi^{B}$ increases, consistent with Corollary 1 . The top-right panel demonstrates that the classic Cournot distortion increases on the worker-side and decreases on the firm-side as $\phi^{B}$ increases, which is consistent with a decreasing inverse hazard rate. The bottom-left panel of Figure 1 shows that the Spence distortion is always positive for workers - i.e. the low-price side of the market, and always negative for firms i.e. the high-price side of the market. 
The market distortion as a whole on each side of the market is the sum of the Cournot and Spence distortions, given in the top-right and bottom-left panels of Figure 1 respectively. The total market distortion also is the difference between the private and Pigouvian prices, given in the bottom-right panel of Figure 1 for workers and for firms. Note that in this example the Pigouvian platform always subsidizes the more efficient worker-side of the market and recovers this subsidy from the firm-side, consistent with Proposition 2 for a constant returns to scale matching function.

The bottom-right panel further implies that the total market distortion on the workerside is unambiguously upward, even though the worker side is the low-priced side of the market. The private price for firms is smaller than their Pigouvian price for minimal asymmetry in the matching technology. In other words, despite that the firm-side is the high priced side of the market, the private price is still too low compared to what is socially optimal. Together with the positive total market distortion on the worker-side of the platform, this implies that the inequality in prices between sides of the platform is too low from a social point of view.

\section{Conclusions}

In this paper we have shown how a private monopoly matchmaker internalizes the matching externalities - a negative own-side congestion and positive cross-side externality - that are inherent to its matching technology. If the matching technology is more efficient (i.e. the negative own-side externality is small and the positive cross-side externality is large) on a side of the market, the monopoly matchmaker will charge that side a lower price for its service. Comparing prices between sides of the platform, we have also shown that a monopoly matchmaker will charge a lower price to the side that matches its users more efficiently. For example, many online listing platforms, such as Monster.com or Forsalebyowner.com, charge sellers that list prices for their goods on the platform a larger

fee than buyers to search among the listed goods, and our model explains why this is the case: a monopoly matchmaker that uses a listing technology can more efficiently match buyers than sellers. 
Our model also predicts that a monopoly matchmaker will only partially internalize the platform's matching externalities. In particular, the Spence distortion on a side of the market is increasing in the platform's matching efficiency for that side of the market. Comparing distortions between sides of the platform, we have also shown that the Spence distortion must be negative (positive) on at least one side of the market if the matching function exhibits decreasing or constant (increasing) returns to scale. For example, assuming that buyers are matched more efficiently in a constant returns to scale matching technology and that heterogeneity in user type is lognormally distributed using realistic parameter values, we have shown that prices are too high for buyers and too low for sellers for minimal asymmetries in matching elasticities between sides of the market. That is, inequality in market prices between sides of the online listing platform is too low from a social point of view.

The economics profession is far from a full understanding of user heterogeneity and sorting, price formation and distortions in markets that increasingly rely on technology to generate trade through matchmaking platforms. This paper may serve to put a few puzzle pieces into their rightful locations. If so, this will mark progress towards a worthy goal. 


\section{Appendix A: Participation fees, per-match fees and platform costs}

All results in the main text are expressed in terms of fixed "per-match" fees denoted by $p^{I}$ for side $I=A, B$. However, all results can instead be expressed in terms of fixed "participation fees" denoted by $P^{I}$, which is levied when users enter the platform, by simply substituting for $p^{I}=$ $P^{I} /\left(M / N^{I}\right)$ in all expressions.

From the point of view of users, the indifference between paying a fixed per-match fee or a fixed participation fee directly follows from the assumptions of risk-neutrality, that matching is random (i.e. match probabilities are the same for users on a particular side) and that there are no transfers between matched users. To illustrate this, note that expected net utility of users when the platform can charge both types of fees is given by:

$$
u^{I}=\left(v^{I}-p^{I}\right) m^{I}-P^{I}
$$

Users participate when $v^{I} \geq \widetilde{v^{I}} \equiv P^{I} / m^{I}+p^{I}$ and demand is given by $N^{I}=n^{I}\left(1-F^{I}\left(\widetilde{v^{I}}\right)\right)$. So, the platform can reach any desired allocation $\left(N^{A}, N^{B}\right)$ whether one or both pricing instruments are available and is therefore indifferent between both.

That the platform is indifferent between fixed per-match and fixed participation fees only holds when platform costs are independent of the type of fee charged. To see this, assume that the platform incurs a per-match cost $c$ proportional to the amount of matches that occur through the platform and a side-specific cost $C^{I}$ to attract users on each side. When both fees are available to the platform, profits can be written as:

$$
\pi=\left(p^{A}+p^{B}-c\right) M\left(N^{A}, N^{B}\right)+\left(P^{A}-C^{A}\right) N^{A}+\left(P^{B}-C^{B}\right) N^{B}
$$

When $c, C^{A}$ and $C^{B}$ are constants, the platform can maximize profits by choosing the optimal allocation $N^{A}, N^{B}$ and by setting both types of fees such that the right amount of users on each side are attracted. Note that any allocation can be reached by setting $p^{A}=p^{B}=0$ and adjusting the participation fee or by setting $P^{A}=P^{B}=0$ and adjusting the per-match fee. But when $c, C^{A}$ and $C^{B}$ are not constant and depend on the value of the fees charged, the equivalence between the two fee types no longer holds. For example, if $c=0$ when $p^{A}=p^{B}=0$ and $c>0$ when $p^{A}$ or $p^{B}>0$ and $C^{A}$ and $C^{B}$ constant, then the platform always prefers participation over flat fees. 
This scenario is particularly relevant when it is costly for matchmakers to observe individual matches in order to charge fixed per-match fees. ${ }^{8}$

Finally, note that the discussion so far assumed that the availability of a single pricing instrument on each side of the market suffices for the platform to reach its desired allocation. However, the presence of network externalities typically implies that the platform faces a problem of equilibrium multiplicity. As suggested by Caillaud and Jullien (2001), the availability of multiple pricing instruments on each side of the market can help the platform to reach its preferred equilibrium. This issue is further addressed in Appendix B.

\footnotetext{
${ }^{8}$ When the platform observes matches, it can typically do better than charging fixed per-match fees. When (part of) the match surplus is observed, the platform can price discriminate between users. For example, real estate brokers observe the selling price of a transaction or temporary help agencies observe the wage of the workers they assign to firms. These platforms typically price discriminate users by charging a commission fee proportional to the observed component of the match surplus. In our model, fixed per-match fees and proportional fees are not equivalent.
} 


\section{Appendix B: Equilibrium existence and uniqueness}

It is well-known from the platform literature that if a platform chooses optimal prices in the first stage and users of the two sides simultaneously decide on participation in the second stage, there is an inherent problem of equilibrium multiplicity due to user coordination failures. Borrowing the terminology in Caillaud and Jullien (2001), a "bad-expectation” market allocation can always prevail in which case none of the users of either side participate, whatever prices charged by the platform. This because they have negative beliefs about the participation decision of users on the other side. So, the question is when the "good-expectation" market allocation will prevail that was described in the main text of the paper.

One way to address the issue is to assume a "rational-expectations" equilibrium as suggested by Caillaud and Jullien (2003). The intuition is that users on both sides have "favorable" beliefs about the participation decision of other users. When there exists an equilibrium that for users of both sides is favorable compared to, for example, the nonparticipation equilibrium, users will decide to participate. In our setting, when users on side $A$ have favorable beliefs about the participation decision of $B$-side users, they participate when their net match valuation is greater than or equal to the per-match fee charged by the platform. So, demand on side $A$ is equal to $N^{A}=n^{A}\left(1-F^{A}\left(p^{A}\right)\right)$. Similarly, under favorable beliefs demand on side $B$ is equal to $N^{B}=n^{B}\left(1-F^{B}\left(p^{B}\right)\right)$ and there exists a unique set of prices that corresponds to the monopolist's profit maximizing allocation. Moreover, as suggested by Caillaud and Jullien (2001), the assumption of favorable beliefs is unnecessary if the platform can use multiple pricing instruments. For example, when the platform can charge per-match as well as participation fees on each side of the market, it can grant a small participation subsidy arbitrarily close to zero such that all users on both sides are willing to participate. The platform can then adjust its per-match fees to establish its profit maximizing allocation. This would also be possible in our model since we have that fixed per-match and participation fees are isomorphic as was discussed in Appendix A,

In case the platform charges participation fees conditional on the amount of users or "insulating tariffs", Weyl (2010) shows how a unique equilibrium can exist. The intuition is that the platform can select any chosen amount of participants on a particular side by charging them a price conditional on the amount of users entering on the other side of the market. For this to be 
possible, there must be a unique price on a side of the market which can be written as a function of participation on both sides. In our model, inverse demand in terms of a fixed participation fee on side $I$ of a market can be written as:

$$
P^{I}\left(N^{I}, N^{J}\right)=F^{I^{-1}}\left(1-\frac{N^{I}}{n^{I}}\right) \frac{M\left(N^{I}, N^{J}\right)}{N^{I}}
$$

Now, note that for any given amount of $J$-side participants, say $\widetilde{N^{J}}$, there is a unique price that pins down or insulates the level of $I$-side participation desired by the platform, say $\widetilde{N^{I}}$, and $P^{I}\left(\widetilde{N^{I}}, \widetilde{N^{J}}\right)$ is the unique insulating tariff. Once the participation rate on side $I$ is fixed there is no longer a coordination problem on side $J$ and the platform can attract any desired amount of users. In other words, the platform can implement any desired allocation $\widetilde{N^{A}}, \widetilde{N^{B}}$ by charging an insulating tariff on at least one side of the market. 


\section{References}

Armstrong, M. 2006. "Competition in Two-Sided Markets" RAND Journal of Economics, Vol. 37, No 3, Autumn 2006, 668-691.

Belleflamme, P. and Toulemonde, E. 2009. "Negative Intra-Group Network Externalities In Two-Sided Markets" International Economic Review, Vol. 50, No. 1, February 2009.

Caillaud, B. and Jullien, B. 2001. “Competing Cybermediaries." European Economic Review, 45(4 - 6): 797 - 808.

Caillaud, B. and Jullien, B. 2003. "Chicken and Egg: Competition among Intermediation Service Providers" RAND Journal of Economics, Vol. 34, No 2, Summer 2003, 309-328.

Chen, K-P. and Huang, Y-C. 2012. "A Search-Matching Model of the Buyer-Seller Platforms" CESifo Economic Studies, 2012, doi:10.1093/cesifo/ifs032.

Cournot, A. 1838. "Researches into the Mathematical Principles of the Theory of Wealth" (N.T. Bacon trans.), 1971 edition. Augustus M. Kelley, New York.

Damiano, E. and Li, H. 2007. "Price Discrimination and Efficient Matching" Economic Theory, Vol. 30, pp. 243-263.

Damiano, E. and Li, H. 2008. "Competing Matchmaking" Journal of the European Economic Association, Vol. 6, pp. 789-818.

Ellison, G. and Fudenberg, D. 2003. "Knife-Edge or Plateau: When Do Market Models Tip?" Quarterly Journal of Economics, 118(4): 1249--78.

Fabinger, M. and Weyl, G. 2012. "Pass-Through and Demand Forms" Working paper.

Niedermayer, A. and Shneyerov, A. 2013. "For-Profit Search Platforms" Forthcoming in the International Economic Review.

Petrongolo, B. and Pissarides C. 2001. "Looking into the Black Box: A Survey of the Matching Function" Journal of Economic Literature, Vol. XXXIX (June 2001), 390--431.

Rochet, J-C. and Tirole, J. 2003. "Platform Competition in Two-Sided Markets" Journal of the European Economic Association, Vol. 1, 990-1029.

Rochet, J-C. and Tirole, J. 2006. "Two-Sided Markets: A Progress Report" RAND Journal of Economics, Vol. 37, No 3, Autumn 2006, 645-667.

Spence, M.A. 1975. "Monopoly, Quality, and Regulation" The Bell Journal of Economics, 6, 417--429.

Weyl, G. 2010. "A Price Theory of Multi-Sided Platforms" American Economic Review 100 (September 2010), 1642-1672. 
Figure 1: Optimal Prices and Distortions on Both Sides of the Market
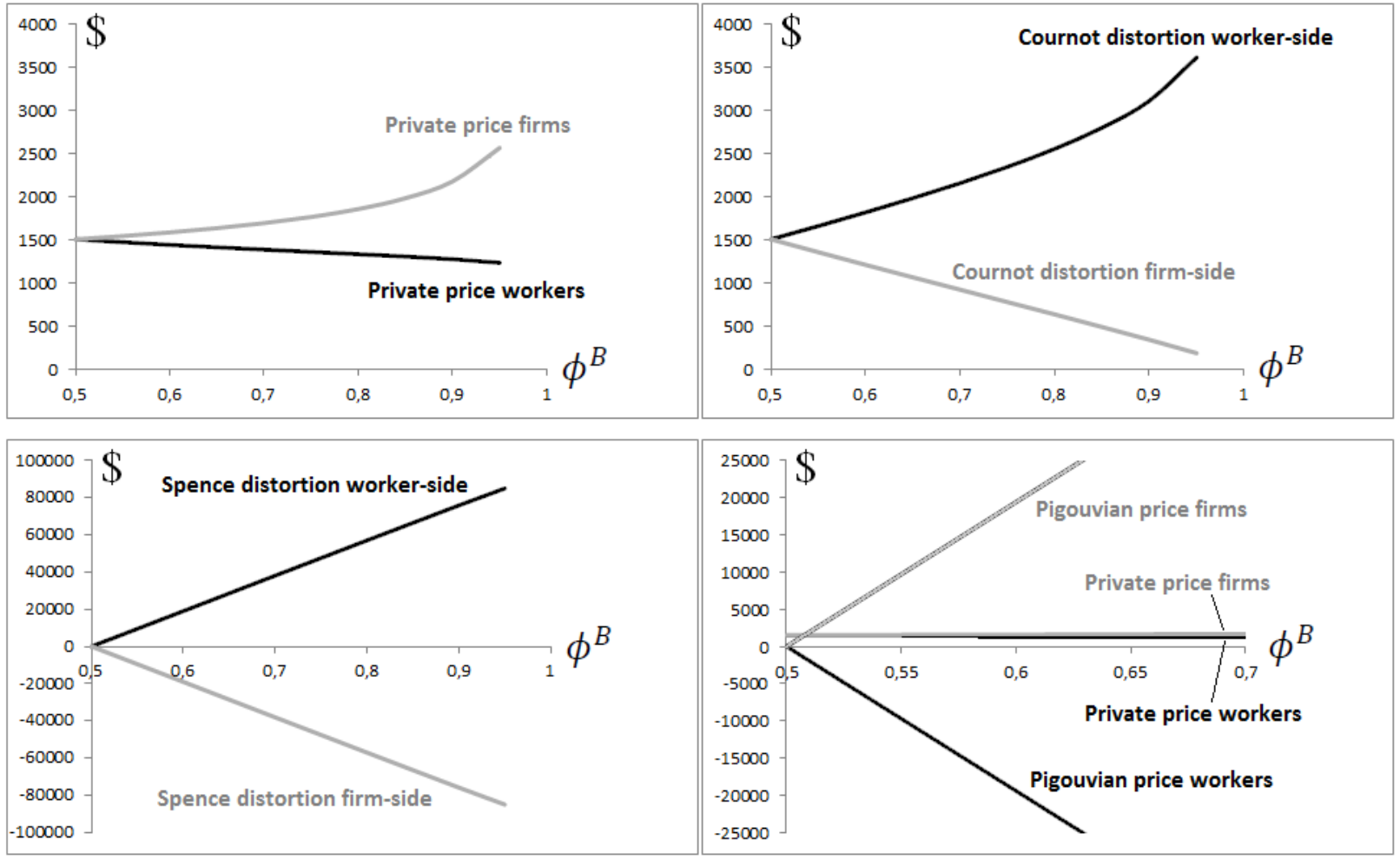